GRADIATION\&APPLICATIONS

ISSN 2466-4294 (online) | rad-journal.org

Vol. 2 | Issue 2 | pp. $75-81,2017$

doi: 10.21175/RadJ.2017.02.017

Original research paper

\title{
DEVELOPMENT OF PEDIATRIC MODEL OF HEMATOPOIETIC ACUTE RADIATION SYNDROME (H-ARS) AND COUNTERMEASURE TESTING USING THE GÖTTINGEN MINIPIG*
}

\author{
Amandeep Kaur ${ }^{1}$, Nagalaxmi Vemalapally ${ }^{1}$, Grant Severson', \\ Jatinder Gulani ${ }^{2}$, David Bolduc ${ }^{1}$, Maria Moroni ${ }^{* *}$ \\ ${ }^{1}$ Armed Forces Radiobiology Research Institute, USUHS, Bethesda, MD, USA \\ ${ }^{2}$ National Cancer Institute, NIH, Frederick, MD, USA
}

\begin{abstract}
There is a pressing need to develop animal models as well as treatment appropriate for age-specific radiation injuries. The minipig represents a promising animal model for testing the effects of radiation on the pediatric population. We subjected piglets, age 6 weeks old (corresponding to less than 2 years old in human), to either sham irradiation or to total body irradiation ( ${ }^{60}$ Cobalt $0.6 \mathrm{~Gy} / \mathrm{min}$ ) at hematopoietic doses spanning from 1.6 Gy to $2.0 \mathrm{~Gy}$, and determined the dose-survival relationship and course of radiation injury in the presence of minimal supportive care. The LD5o/45 was determined to be $1.83 \mathrm{~Gy}$ [CI 1.70 - 1.91]. The course of hematopoietic acute radiation syndrome $(H-A R S)$ in the piglet model resembled that of humans, with four distinct phases namely, prodromal phase, latent phase, manifest illness phase, and recovery or death. Kinetics of blood cell loss such as sudden lymphopenia, decline in neutrophil counts preceded by initial granulocytosis, erythrocytopenia, and thrombocytopenia with a characteristic shoulder followed by partial recovery mimicked the expected radiationinduced changes. Moribund animals were characterized by anorexia, lethargy, fever or hypothermia, bleeding, and dyspnea. Upon euthanasia, animals displayed dose dependent bone marrow hypoplasia and hemorrhages in several organs. Granulocyte colony stimulating factor (G-CSF), a countermeasure approved for H-ARS in humans and effective in adult minipig, was tested in the piglets. Administration of G-CSF enhanced survival by $37.5 \%$ and reduced both duration as well as nadir of neutropenia. In conclusion, the minipig provides a practical and feasible animal model for H-ARS and development of radiation countermeasures for the pediatric population.
\end{abstract}

Key words: Animal models, countermeasure testing, G-CSF, hematopoietic acute radiation syndrome, minipig, pediatric, total body irradiation

\section{INTRODUCTION}

Both adult and pediatric animal models for the characterization of the hematopoietic acute radiation syndrome (H-ARS) and drug development are urgently required. Under the FDA Animal Rule, data for radiation countermeasure efficacy testing can be extrapolated from animal models [1]. Nevertheless, drugs' efficacy and safety data cannot be directly translated from adult to pediatric animal models, due to major differences in the organ anatomy, maturation stage, physiology and metabolism between adults and children affecting radio-sensitivity, pharmacokinetics and pharmacodynamics, drug toxicity, disposition and action [2]. Therefore, countermeasures shown to provide benefits to adults must be tested in animal models of an age range with the same developmental characteristics as the target population.

The minipig represents a promising tool to study the influence of age on susceptibility to radiation insult. Benefits include short reproductive cycle, prolificacy, large litter size, ease of cross-fostering, similarities to the human pediatric population in organ development, maturation, physiology and anatomy, as well as multiple administration routes (sub-cutaneous, intramuscular, intra-dermal, and intra-venous) to test intact drug formulation for more immediate translation to humans. Young minipigs have been used in the past as models for studying diseases in children. Juvenile minipigs were used as a model for hemorrhagic hypovolemic shock, lung injury, hemodynamics, cardiovascular pathophysiology, and diabetes [3]. Pediatric Chinese minipigs were successfully used to develop a hemodynamically stable model of acute lung injury $[4,5]$.

Our previous data on irradiated minipigs confirm that this model provides a suitable system to test radiation countermeasures and to study the course of ARS progression [6-11]. In light of the longer life expectancy and fast growth rate, children are of special concern in radiation protection [12]. Here we focused on the characterization of a pediatric model of H-ARS, using the Göttingen minipig irradiated at age corresponding to infant and children $<2$ years of age,

\footnotetext{
* The paper was presented at the Fifth International Conference on Radiation and Applications in Various Fields of Research (RAD 2017), Budva, Montenegro, 2017.

maria.moroni@usuhs.edu
} 
A. Kaur et al., Development of pediatric model..., Rad. Applic., 2017, 2, 2, 75-81

as a surrogate to mimic the human condition. The longevity of minipigs (12 years) is closer to that of humans relative to smaller animals (e.g. rodents), thus facilitating comparison of age-ranges between humans and the minipig model.

\section{MATERIALS AND METHODS}

\subsection{Animals, housing and feeding}

Sixty-four 5 -week old male Göttingen minipigs ( $n=36$ for survival study; $n=28$ for G-CSF study) were obtained from Marshall BioResources (North Rose, NY). All animal procedures were approved by the Armed Forces Radiobiology Research Institute (AFRRI) Institutional Animal Care and Use Committee (IACUC). AFRRI is fully accredited by the Association for Assessment and Accreditation of Laboratory Animal Care, International. All procedures were consistent with the Guide for the Care and Use of Laboratory Animals [13] and AFRRI animal facility's standard operating procedures. Animals were anesthetized at the time of irradiation, blood collection, and euthanasia in order to minimize potential pain and distress associated with these procedures.

Animals were group-housed at their arrival and were moved to single cages 4-6 days before irradiation. This was done to avoid introducing the stress of separation and irradiation at the same time. After irradiation, animals remained in single cages for monitoring food intake and preventing injuries from pushing and biting, a normal behavioral trait in social animals while establishing a hierarchy. Food was provided twice a day in the form of dry pig chow (Laboratory minipig starter chow, PMI Nutrition Intl., LLC, Brentwood, MO) and swine milk formula (Solustart II pig milk replacer, Land O Lakes Inc., Arden Hills, MN; $120 \mathrm{ml}$, total daily). Water was offered ad libitum. Rooms were maintained on a 12:12 $\mathrm{h}$ light dark cycle. All piglets were provided with a heating pad for warmth.

\subsection{Irradiation}

A total of 64 animals were irradiated for this study ( $\mathrm{n}=36$ for survival study; $\mathrm{n}=28$ for G-CSF study, including sham irradiated). The dose of irradiation ranged between 1.6 Gy to 2.0 Gy. Piglets were anesthetized with Telazol $\AA \quad(4-6 \quad \mathrm{mg} / \mathrm{kg})$ and isoflurane, and were given ${ }^{60} \mathrm{Co}-$ gamma-photons delivered bilaterally at $0.6 \mathrm{~Gy} / \mathrm{min}$, TBI, as described in a previous publication [8]. The prescribed dose was delivered to the midline tissue at the widest crosssection of the abdomen, measured with a caliper while the anesthetized animal was lying in a Panepinto sling. Real-time dosimetry was performed during the irradiations with an ionization chamber as a quality control check. The day of irradiation was considered day o for blood sampling. Animals were followed for 45 days after irradiation.

\subsection{Blood sampling, indicators of health, and pathology}

Blood samples were obtained from the saphenous and/or cephalic veins after anesthetizing the animals 76 with isoflurane via face mask (induction at $5 \%$ and maintenance at 2-3\%); right and left limbs were used alternately. Blood samples were collected once before irradiation followed by days $0,1,3,7,10,14,17,20,23$, $28,35,42$, and 45 post-irradiation. Blood was collected in EDTA tubes and immediately processed for complete blood counts with differentials (CBC/diffs) using the ADVIA 2120 (Siemens Medical Solutions Diagnostics, Ireland). For the purpose of this study, neutropenia will be referred to ANC $<500 / \mu \mathrm{l}$ and thrombocytopenia will be referred to platelet counts $<20,000 / \mu \mathrm{l}$. Indicators of health (activity, posture, anorexia, stool consistency, respiration rate and quality, vomiting, body temperature, lung sound, heart rate and rhythm, pulse quality, hydration status, abdominal distention, presence of petechiae and frank blood) were recorded during the 45-day time period. Blood samples for $\mathrm{CBC} /$ diffs were also taken from adult minipigs (3 and 6 months old, $n=43$ and 51, respectively) that were exposed to total body irradiation at same radiation doses (1.6-2.0 Gy) and euthanized at day 30 post-irradiation.

\subsection{Supportive care}

Minimal supportive care was given in the form of nutritional support and antibiotics, from day o (day of irradiation) to the end of study (day 45). Analgesia (buprenorphine) was provided as necessary, for prevention of pain. Nutritional support and dietary enrichment consisted of salty and sweet treats, yogurt, probiotics, omega-3 fatty acid supplements, metamucil, and pedialyte. A prophylactic antibiotic regimen was chosen based on guidelines from the American Society of Cancer Oncology, the Department of Health and Human Services, Radiation emergency medical management, and the Radiation Injury Treatment Network. A number of micro-organisms had been previously identified in the blood and tissues of irradiated Göttingen minipigs [6], which justifies the use of broad spectrum antibiotics for a prophylactic empirical antibiotic regimen. Ciprofloxacin $(5 \mathrm{mg} / \mathrm{kg}$, BID, PO) plus amoxicillin/clavulanate (11 mg/kg, BID, PO) were given orally once absolute neutrophil counts (ANC) reached $<500 / \mu$, or was expected to drop below $500 / \mu \mathrm{l}$ within 48 hours. Ciprofloxacin is a fluoroquinolone agent with antibiotic activity against a broad spectrum of Gram-negative and Gram-positive bacteria responsible for infections to the respiratory, urinary and gastrointestinal tract. Fluoroquinolones (ciprofloxacin and levofloxacin) are widely used in the treatment of human disease and are likely to be used in the event of radiation exposure to prevent infections [14]. Levofloxacin may cause problems with bones, joints, and tissues around joints in children, and it is not recommended for children. This was the reason why we opted to use ciprofloxacin for the pediatric model. Amoxicillin/clavulanate is used to treat mild-tomoderate bacterial infections including sinusitis, bronchitis, and otitis media and is the recommended antibiotic to be used in combination with fluoroquinolones for empiric therapy regimen [15]. Antibiotics were withheld once the ANC returned to $500 / \mu l$, and remained above that level for 48 hours. 


\subsection{G-CSF administration}

Dose of G-CSF was chosen based on a pilot experiment using 12 sham-irradiated piglets that received either $5 \%$ dextrose $(n=3)$ or $2.5,5$, and 10 $\mu \mathrm{g} / \mathrm{kg}$ G-CSF ( $\mathrm{n}=3$ /group). We then subjected 16 Göttingen minipigs, 6 weeks age, to the LD70/45 (1.92 $\mathrm{Gy},{ }^{60} \mathrm{Co}$ - gamma-photons, $0.6 \mathrm{~Gy} / \mathrm{min}$, TBI). Animals were administered daily either $5 \mu \mathrm{g} / \mathrm{kg} \mathrm{G}-\mathrm{CSF}(\mathrm{n}=8)$ or $5 \%$ dextrose $(\mathrm{n}=8)$ in equal volume. Drug administration continued until ANC reached $1000 / \mu \mathrm{l}$ for three consecutive blood draws, but not to exceed day 29 post-irradiation. Blood samples were collected once before irradiation and twice a week afterwards.

\subsection{Necropsy and euthanasia criteria}

Animals were euthanized according to current AVMA guidelines [16]. The minipigs were sedated with Telazol@ (6-8 mg/kg IM) and/or isoflurane and injected intra-venous or intra-cardiac Euthasol@ (sodium pentobarbital, $1 \mathrm{ml} / 4.5 \mathrm{~kg}$ ). Death was confirmed via auscultation of the heart and cessation of sounds for at least 5 minutes. Criteria for humane euthanasia before completion of the survival study were divided into absolute and non-absolute. Absolute criteria: non-responsiveness, prolonged dyspnea, loss of $20 \%$ body weight, and hypothermia $\left(<36^{\circ} \mathrm{C}\right)$. Nonabsolute: hyperthermia $\left(>41^{\circ} \mathrm{C}\right)$, complete anorexia for $>18$ hours, anemia/pallor, CRT >2 sec, petechiation and ecchymosis in association with severe thrombocytopenia $(<20,000 / \mu \mathrm{l})$, vomiting, diarrhea, seizure, vestibular signs, prolonged hemorrhage, inability or extreme reluctance to stand, or other signs of severe organ system dysfunction with a poor prognosis. Non-absolute criteria were used for euthanasia when animals did not meet absolute criteria but deteriorated rapidly in condition due to various combinations of non-absolute criteria. Necropsy was performed on all euthanized animals; histopathological examination of hematoxylin and eosin (H\&E) stained tissue slides was conducted by a board certified pathologist.

\subsection{Statistical analysis}

Basic descriptive analysis, one or two-sided Student's t-tests were done in Excel or PASW Statistic 18 software, SPSS Inc, IL, USA. Probit regression analysis was used to generate the probit curve, using the PASW Statistic 18 software, SPSS Inc, IL, USA. Survival curves were plotted using the Kaplan-Meier method.

\section{RESULTS}

\subsection{Probit curve and natural history}

Survival of piglets irradiated at doses spanning from 1.6 to 2.0 Gy was followed for 45 days. The day of irradiation and following time period, animals displayed partial anorexia and lethargy which lasted for 5-7 days (prodromal phase). Afterwards, animals were alert and responsive (latent phase) unless they became sick, in which case they alternated periods of activity with periods of inertia. Piglets displayed the first overt sign of H-ARS in the form of varying degrees of petechial hemorrhages around day 13 post TBI. Petechiae ranged from pinpoint to 1-2 $\mathrm{mm}$ in diameter. Sickness was characterized by long periods of inactivity, abnormal posture, abnormal stool consistency, anorexia, dehydration, poor perfusion (CRT >2 sec), labored breathing, and hemorrhages in the form of petechiae, bruising, and/or blood in stool, urine or body openings (manifest illness phase). Antibiotics were instituted on day 14 post TBI (+/- 3.5 days, in alignment with the onset of neutropenia). The median duration of the antibiotic treatment was 20 days in animals that recovered from neutropenia. Antibiotic-induced loose stool or mild diarrhea was observed in 15 test animals which was mitigated by providing probiotics, yogurt, pedialyte, and fruits. Abnormal stool was also seen in the form of hard stool which was treated with metamucil. At the time of euthanasia, neurologic signs (ataxia, loss of righting reflex, head tilt, and circling), external bleeding (eyes, nostrils, or mouth), fever or hypothermia, bloat, and hematuria were also observed in some cases.

The relationship between irradiation dose and survival over a period of 45 days is reported in Figure 1. Probit analysis was used to fit survival curves and calculate dose-response. The LD50/45 (Figure 1) was calculated as $1.83 \mathrm{~Gy}$ [CI $1.70-1.91]$. The values for LD30/45, LD70/45, and LD90/45 were $1.74 \mathrm{~Gy}$ [CI 1.45-1.82], 1.92 Gy [CI 1.85-2.12] and 2.05 Gy [CI 1.95$2.52]$, respectively.

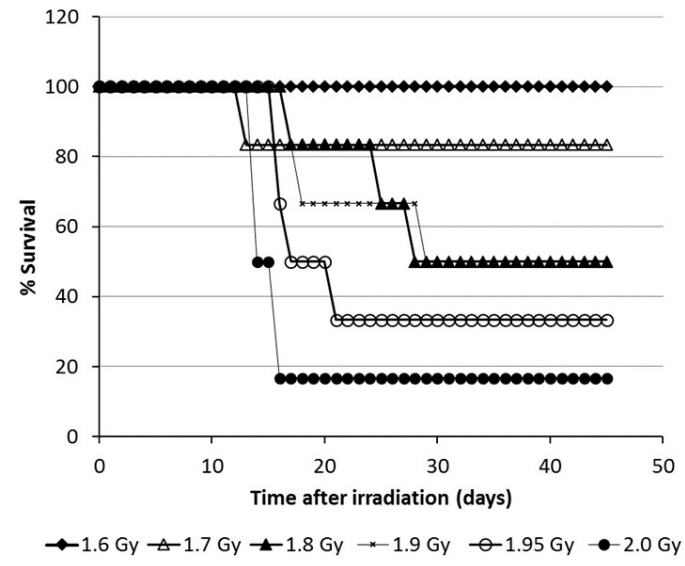

Figure 1. Dose response curves for bilateral gammairradiation of pediatric Göttingen minipig. Six animals per dose were irradiated with Cobalt-6o (o.6 Gy/min). Survival was followed for 45 days after exposure and data were plotted as Kaplan-Meier curves.

Gross examination of animals, euthanized between day 13 and 28 post-irradiation, revealed mild to severe multifocal and/or multifocal coalescing petechiae in stomach, small and large intestines; multifocal petechiae and ecchymoses in urinary bladder, gall bladder, kidneys, dura mater and brain. All lymph nodes were enlarged and diffusely hemorrhagic for non-surviving animals. Cerebellar hemorrhage was in most instances associated with ataxia. Areas of cardiac infarct were seen in a minority of animals. Higher prevalence of cardiac hemorrhages (13/16 versus $2 / 16$ ) 
A. Kaur et al., Development of pediatric model..., Rad. Applic., 2017, 2, 2, 75-81

and pulmonary hemorrhages (12/16 versus $0 / 16)$ in non-survivors vs survivors was associated with lethality. After irradiation, bone marrow in the sternum was depleted in a dose-dependent fashion, as determined by percent cellularity in the sternal marrow. None of the animals irradiated with $1.6 \mathrm{~Gy}$ had signs of bone marrow depletion. Among animals irradiated at higher doses, depletion of bone marrow cellularity was observed in $50 \%$ of minipigs receiving $1.7-1.8 \mathrm{~Gy}$, and in $100 \%$ of the animals irradiated with 1.9 - 2.0 Gy. Decedent animals showed marked to severe bone marrow depletion $(\sim 40-50 \%$ to $<5 \%$ marrow cells) with increasing radiation doses. Survivors had mild decrease in cellularity $(\sim 60-80 \%$ marrow cells to normal marrow), irrespective of the radiation dose. Bone marrow cellularity in control, sham-irradiated animals was $\sim 80-90 \%$ marrow cells, with $10-20 \%$ adipose tissue.

\subsection{Kinetics of blood cell decline and recovery}
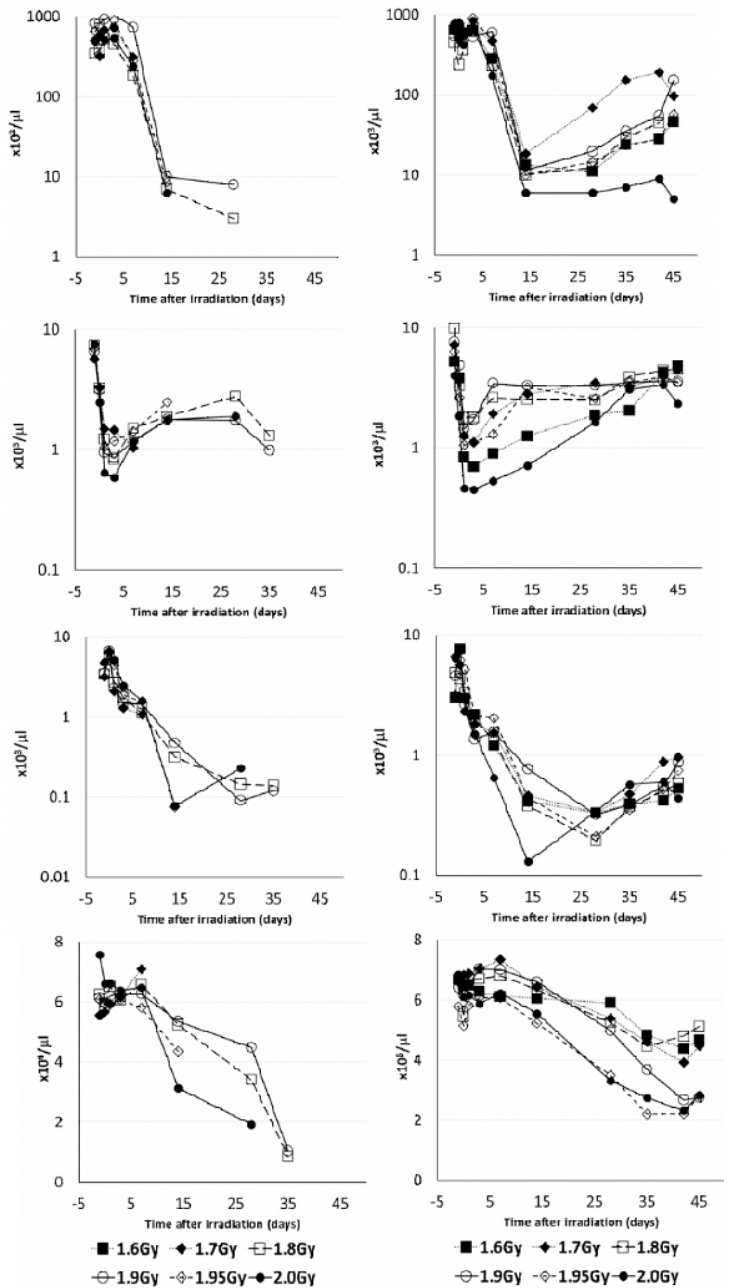

Figure 2. Blood count patterns by survival status (decedents: left; survivors: right) and radiation dose. Mean values for platelets, lymphocytes, neutrophils, and RBCs (top to bottom panels) are shown.
After irradiation, blood counts for most parameters showed the expected kinetics of cell loss and recovery (Figure 2). Lymphocytes declined quickly, ANCs were characterized by initial granulocytosis followed by decline and a partial recovery, and platelets exhibited the characteristic shoulder followed by decline and a partial recovery. RBC loss was more severe and occurred faster in lethally irradiated animals. On average, all animals (survivors and decedents) developed neutropenia (ANC $<500 / \mu \mathrm{l}$ ) and thrombocytopenia (platelets $<20,000 / \mu \mathrm{l}$ ) around day 14 post-irradiation. Prevalence and duration of neutropenia and thrombocytopenia were dosedependent, and lasted for 2 to 4 weeks. Full recovery was not reached within the 45 -day observation period.

We next compared CBC counts in pediatrics with respect to older animals (3 and 6 months old) (Table 1). Before irradiation, lymphocyte, neutrophils and platelet counts were higher in the younger animals $(\mathrm{p} \leq$ o.01), in contrast to the RBCs, Hgb and HCT. After irradiation, RBCs, Hgb and HCT counts at euthanasia in lethally irradiated piglets were significantly lower with respect to lethally irradiated adults. However, there were no differences seen among the pediatric and adult survivors.

Table 1. Comparison of CBC counts by age in Göttingen minipigs, before irradiation and at euthanasia

\begin{tabular}{|c|c|c|c|c|c|c|c|}
\hline & $\stackrel{\infty}{\infty}$ & 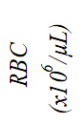 & 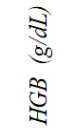 & $\frac{2}{2}$ & 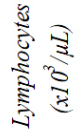 & 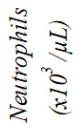 & 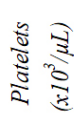 \\
\hline \multirow{3}{*}{$\begin{array}{c}\text { Pre- } \\
\text { Irradiation }\end{array}$} & $5 \mathrm{wk}$ & 6.5 & 11.11 & 34.23 & $7.05 \%$ & $4.36^{*}$ & $596^{*}$ \\
\hline & $3 \mathrm{mo}$ & 7.79 & 1.8 & 37.19 & 4.03 & 3.53 & 491 \\
\hline & $6 \mathrm{mo}$ & 8.14 & 13.05 & 38.77 & 3.67 & 3.27 & 408 \\
\hline \multirow{3}{*}{$\begin{array}{r}\text { Euthanasia } \\
\text { (decedents) }\end{array}$} & $5 \mathrm{wk}$ & $1.54^{*}$ & $2.61 *$ & $8.07 *$ & 1.22 & 0.08 & 4 \\
\hline & $3 \mathrm{mo}$ & 2.7 & 4.33 & 13.14 & 0.65 & 0.07 & 4 \\
\hline & $6 \mathrm{mo}$ & 2.43 & 4.19 & 12.24 & 0.4 & 0.05 & 3 \\
\hline \multirow{3}{*}{$\begin{array}{l}\text { Euthanasia } \\
\text { (survivors) }\end{array}$} & $5 \mathrm{wk}$ & 4.3 & 8.76 & 27.66 & 4.13 & 1.04 & 102 \\
\hline & $3 \mathrm{mo}$ & 5.63 & 11.31 & 33.72 & 3.38 & 2.09 & 142 \\
\hline & $6 \mathrm{mo}$ & 6.73 & 12.32 & 36.97 & 2.3 & 1.44 & 131 \\
\hline
\end{tabular}

Mean values are shown, $* \mathrm{p} \leq 0.01$

3.3. G-CSF increases neutrophil counts, improves survival, and reduces incidence of neutropenia

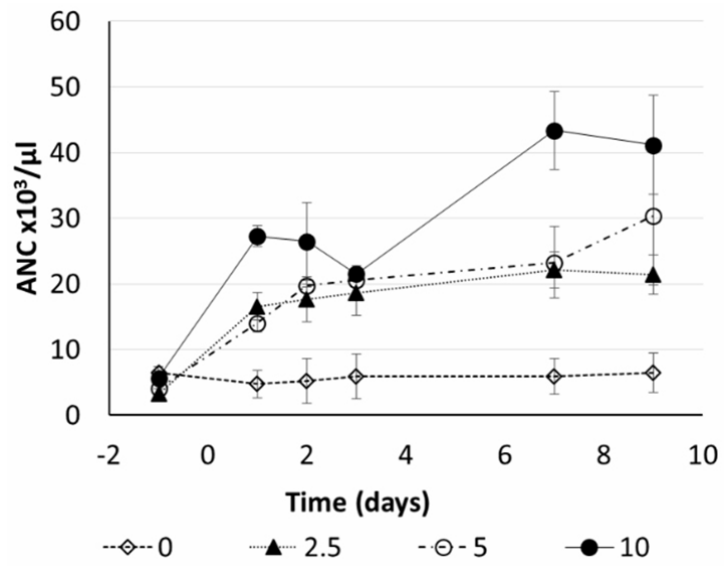

Figure 3. Average of neutrophils (ANC) from sham irradiated animals, administered dextrose (o) or G-CSF at 2.5, 5.0 or 10 (microgram per kilogram) ( $\mathrm{n}=3 /$ treatment) 
Un-irradiated animals were administered for 9 consecutive days, subcutaneously, either G-CSF at a concentration of either $2.5,5$ or $10 \mu \mathrm{g} / \mathrm{kg} /$ day $(\mathrm{n}=3$ animals per drug concentration), or dextrose as control. G-CSF elevated neutrophil counts at all doses tested in comparison to age matched control treated with dextrose (Figure 3).

The dose of $5 \mu \mathrm{g} / \mathrm{kg} /$ day was chosen for the survival study, as it is the dose provided to children in the clinic. Efficacy of G-CSF to improve survival, blood cytopenia and bone marrow recovery was assessed using 16 minipigs irradiated at $1.92 \mathrm{~Gy}(\mathrm{LD} 70 / 45)$ and randomly divided into control $(\mathrm{n}=8)$ and $\mathrm{G}-\mathrm{CSF}$ treated $(n=8)$ groups. Animals were followed for 45 days. Administration of G-CSF increased survival by $37.5 \%$ with respect to dextrose treated animals $(\mathrm{p}=0.315)($ Figure 4$)$.

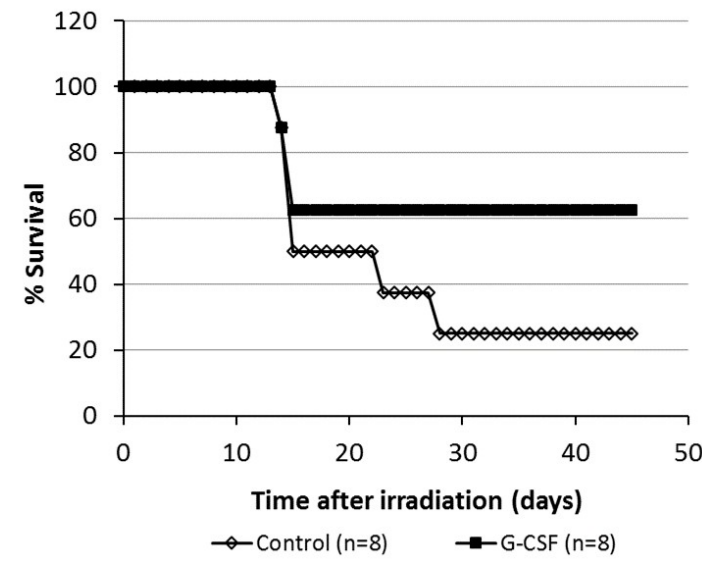

Figure 4. Survival curve after estimated LD70/45 TBI dose $\left({ }^{60} \mathrm{Co}, 0.6 \mathrm{~Gy} / \mathrm{min}\right)$, in minipigs receiving granulocyte colony stimulating factor (G-CSF) or dextrose, starting 24 hours after irradiation ( $\mathrm{n}=8$ animals/group)

Five out of 8 (62.5\%) animals survived in G-CSF treated animals, versus $2 / 8$ survived (25\%) in control treated animals. Increased survival was associated with higher bone marrow cellularity. In the G-CSF treated group, $5 / 8$ animals had bone marrow cellularity ranging from $60 \%$ to $>90 \%$, while $3 / 8$ animals had $<30 \%$ cellularity. On the contrary, in the dextrose treated group, $6 / 8$ animals had $<30 \%$ cellularity, and $2 / 8$ animals had $60-90 \%$ cellularity.

Neutrophil counts and neutropenia were compared between control and treatment groups (Figure 5). ANC counts were increased in the G-CSF group. All decedent animals were neutropenic, regardless of treatment. Within the decedents G-CSF group, neutropenia occurred sooner (day 10-14) with respect to dextrose controls (day 14-23). Among survivors, only 33\% (2/6 animals) were neutropenic in the G-CSF group, with respect to $100 \%(2 / 2)$ in the control group. Recovery was still incomplete by day 45 after irradiation. Platelet decline was similar among dextrose and G-CSF treated group; platelets recovery was not statistically different between the two groups.
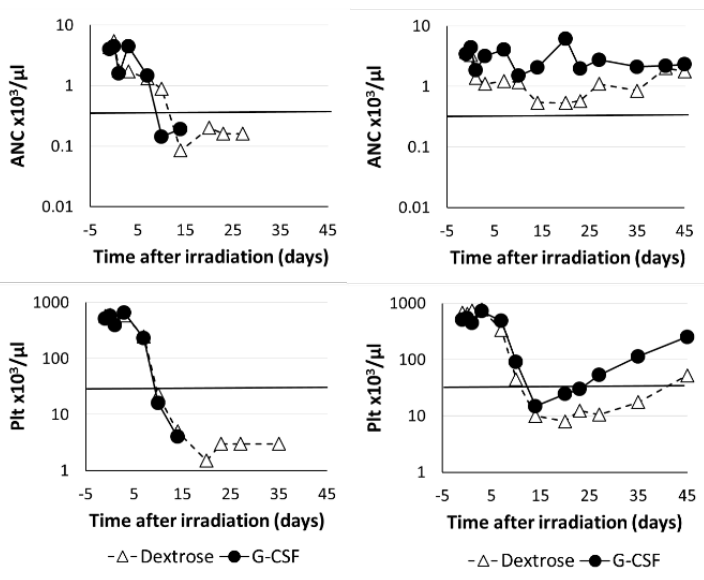

Figure 5. Effect of G-CSF administration on absolute neutrophil count (ANC) and platelets in irradiated minipigs (1.92 Gy, TBI, ${ }^{60} \mathrm{Co}, 0.6 \mathrm{~Gy} / \mathrm{min}$ ) (decedents: left; survivors: right). Solid lines indicates threshold for cytopenia.

\section{DisCUSSION}

Pediatric animal models are urgently required for development and testing of pediatric-friendly drugs for H-ARS. We subjected piglets (6 weeks of age, corresponding to human 1 month to 2 years) to irradiation and determined the dose-survival relationship, course of radiation sickness and hematology profiles. Furthermore, we administered G$\mathrm{CSF}$, the only FDA approved radiation countermeasure for the H-ARS, to validate the translational potential of the model to humans. Our results show that piglets display dose-dependent survival curves, expected kinetics of blood cell loss, and positive response to G$\mathrm{CSF}$ as a radiation countermeasure. To our knowledge, this is the first attempt to establish a pediatric model of H-ARS using large animals suitable for evaluating potential countermeasures for humans.

As part of the pediatric model characterization, we found that the age at which piglets were used in this study posed a few significant challenges with regards to the consumption of dietary enrichment and oral antibiotics. Normal weaning age for piglets is 4 weeks, when the animal is gradually transitioned to a solid food diet. During the week of acclimation, 5 week old animals were offered a variety of foods (sweet and salty) in addition to pig chow and swine milk replacement. This not only allowed us to determine the food preference of the animals but also accustomed animals to eating different types of foods.

The course of acute radiation injury in the piglets was followed over 45-day period. As per extensive data set available to date, ARS in humans and large animal models is characterized by prodromal, latent and manifest phases. Piglets exhibited all three phases. The prodromal phase in the piglets lasted relatively longer (5-7 days) than that in adult minipigs $(\sim 48 \mathrm{~h})$ [8] and was characterized by partial anorexia, which has not been observed in adults. The manifest phase of H-ARS lasted for about 12-15 days, and culminated with animals exhibiting anorexia, lethargy, labored breathing, and anemia. Piglets were also more likely to 
A. Kaur et al., Development of pediatric model..., Rad. Applic., 2017, 2, 2, 75-81

have neurologic signs such as ataxia, circling, and head tilt at tested radiation doses, with respect to adult minipigs. These clinical signs are rarely seen in adult minipigs in our experience [8]. There were a few instances of sudden death, most likely due to cardiopulmonary insufficiency and/or severe aplastic anemia. Similarly to the adult animals, moribund animals displayed anorexia, lethargy, fever or hypothermia, petechial hemorrhages, bleeding, dyspnea, and occasionally hematuria. Widespread hemorrhages in most organs and dose dependent bone marrow hypoplasia were observed upon euthanasia. Propensity to bleeding at relatively low radiation doses appears to be a main feature of the minipig model, although mice, dogs and non-human primates are also characterized by hemorrhages in most organs, after exposure to lethal doses [17]. The cause of the sensitivity of minipig to acute radiation in terms of relatively low LD50 compared to other species [17] has not been established. In the adult Göttingen minipig, sensitivity was not associated to abnormal response of the bone marrow or the gastrointestinal system $[6,7]$, nor to defects in the DNA repair ability [18], but it appeared to be linked to the extent of systemic inflammation triggered by radiation damage [9]. Features in hormonal status of the Göttingen minipig may contribute to the sensitivity of this strain to irradiation. Indeed, in one report on the use of the adult Göttingen as a model for metabolic syndrome, abnormalities in the growth hormone/insulin-like growth factor 1 (GH/IGF-1) hormone axis, an important mediator of response to systemic inflammatory syndrome, have been suggested [19].

Work on irradiated rodents suggests that age may be a critical factor for acute radiation sensitivity. We did not observe a marked difference in survival of pediatric minipigs with respect to young adult animals [8]. At same radiation doses, the $\mathrm{LD} 5 \mathrm{O} / 45$ in pediatrics $(1.83 \mathrm{~Gy})$ was comparable to LD50/30 of $1.7-1.9 \mathrm{~Gy}$ in minipigs aged 4-5 months [8]. For rodents, evidence from one mouse study using $\mathrm{C}_{57}$ black mice, receiving a single dose of $5.5 \mathrm{~Gy}$, suggests that the lethal effects of irradiation are affected by age at the time of exposure. Mortality was lower in the youngest mice (1day and 15-day old), increased in the intermediate age group (30-day old), and decreased again with age in the older age group [2O]. Other studies with rats confirmed age-dependent variations in the radiosensitivity of the animals [21]. Our results in minipigs suggest that 6 weeks of age, corresponding to human less than 2 years of age, is not within the time frame when the animals are most sensitive to irradiation.

Serial blood sampling in the piglets was conducted to determine differential cell counts during the course of the study. Kinetics of blood cell decline were indicative of lymphopenia, neutropenia, thrombocytopenia and erythrocytopenia. The hematology profile of the irradiated pediatric minipig followed the expected kinetics of cell loss and recovery, although complete recovery of the peripheral blood cell counts was not seen by the end of the study. Patterns of occurrence of cytopenia were similar to what is observed in humans and multiple animal species [7]. CBC counts in healthy pediatric minipig are comparable to that of children, highlighting the potential relevance of this model to human beings. In comparison to young adult minipigs (3 months of age, corresponding to human 12 years and older), piglets had lower blood cell counts in terms of RBCs, $\mathrm{Hg}$, and HCT, while they were higher for neutrophils, lymphocytes and platelets. RBC, Hg, and HCT counts were also significantly lower in lethally irradiated piglets with respect to lethally irradiated adults, suggesting that anemia may play a more significant role among the younger population. This finding is important in light of policy making and national stockpiling for radiological emergencies. Additional studies similar to the present research would better allow for pediatric disaster preparedness and response in case of a national radiological emergency.

We also tested the effect of G-CSF in the pediatric minipig model of H-ARS since G-CSF has proven to be a potent mobilizer of hematopoietic stem cells in children undergoing chemotherapy and radiation therapy [22]. In our study, G-CSF resulted in $62.5 \%$ survival in the test group compared to $25 \%$ survival in the control piglets. This is comparable to $50 \%$ and $78.3 \%$ survival seen in G-CSF-treated adult minipigs at LD70/30 [11] and G-CSF treated NHPs at LD50/60, respectively [23]. G-CSF increased neutrophil counts after $24 \mathrm{~h}$ of administration and kinetics of neutrophil recovery were similar to those observed in pediatric cancer patients with chemotherapy induced neutropenia [24], confirming the potential of the minipig as a model for the human H-ARS.

STUDY LIMITATIONS. The results of this study show that piglets have the potential to be successfully used as a pediatric large animal model for understanding ARS in infants and children, for acute as well as long term studies. The findings were however limited by a relatively small number of animals and a short observation period. Further studies are required to validate the findings of the current work using other large animal models, its relevance to human pediatric population, and the age-related effects of irradiation.

Acknowledgements: Special thanks are due to AFRRI's cobalt facility, veterinary science department, and animal care staff for their dedication to the project and superb animal care. This work was supported by funding from National Institute of Allergy and Infectious Diseases AAI12044-OOOOo50oo.

Disclaimer. The views expressed in this manuscript are those of the author(s) and do not reflect the official policy or position of AFRRI, the Uniformed Services University of Health Sciences, or the Department of Defense.

\section{REFERENCES}

1. P. Aebersold, "FDA experience with medical countermeasures under the animal rule," Adv. Prev. Med., vol. 2012, pp. 1-11, Sep. 2012.

DOI: $10.1155 / 2012 / 507571$

PMid: 21991452

PMCid: PMC3177089

2. Guidance for Industry - Nonclinical Safety Evaluation of Pediatric Drug Products, U.S. Department of Health 
and Human Services Food and Drug Administration, Center for Drug Evaluation and Research, Rockville (MD), USA, 2006

Retrieved from:

http://www.fda.gov/downloads/drugs/guidancecomplia nceregulatoryinformation/guidances/ucm079247.pdf

Retrieved on: Jan. 4, 2017

3. M. M. Swindle et al., "Swine as models in biomedical research and toxicology testing," Vet. Pathol., vol. 49, no. 4, pp. 344-356, Jul. 2012.

DOI: $10.1177 / 0300985811402846$

PMid: 21441112

4. X. Li et al., "A novel and stable "two-hit" acute lung injury model induced by oleic acid in piglets," Acta Vet. Scand., vol. 51, p. 17, Mar. 2009.

DOI: 10.1186/1751-0147-51-17

PMid: 19331663

PMCid: PMC2673213

5. A. J. Liu et al., "Effect of oleic acid-induced acute lung injury and conventional mechanical ventilation on renal function in piglets," Chin. Med. J. (Engl.), vol. 126, no. 13, pp. 2530-2535, Jul. 2013.

PMid: 23823829

6. T. Elliott et al., "Gastrointestinal acute radiation syndrome in Göttingen minipigs (Sus scrofa domestica)," Comp. Med., vol. 64, no. 6, pp. 456-463, Dec. 2014. PMid: 25527026 PMCid: PMC4275081

7. M. Moroni et al., "Hematological changes as prognostic indicators of survival: similarities between Göttingen minipigs, humans, and other large animal models," PLOS ONE, vol. 6, no. 9, p. e25210, Sep. 2011.

DOI: 10.1371/journal.pone.0025210

PMid: 21969873

PMCid: PMC3182184

8. M. Moroni et al., "Hematopoietic radiation syndrome in the Göttingen minipig," Radiat. Res., vol. 176, no. 1, pp. 89-101, 2011.

DOI: $10.1667 / R R 2481.1$

PMid: 21520996

9. M. Moroni et al., "Accelerated hematopoietic syndrome after radiation doses bridging hematopoietic (H-ARS) and gastrointestinal (GI-ARS) acute radiation syndrome: early hematological changes and systemic inflammatory response syndrome in minipig," Int. J. Radiat. Biol., vol. 9o, no. 5, pp. 363-372, May 2014.

DOI: $10.3109 / 09553002.2014 .892226$

PMid:24524283

10. M. Moroni et al., "Significance of bioindicators to predict survival in irradiated minipigs," Health Phys., vol. 106, no. 6, pp. 727-733, Jun. 2014.

DOI: 10.1097/HP.0000000000000109

PMid:24776906

PMCid:PMC4006360

11. M. Moroni et al., "The Gottingen minipig is a model of hematopoietic acute radiation syndrome: G-colony stimulating factor stimulates hematopoiesis and enhances survival from lethal total-body $\gamma$-irradiation," Int. J. Radiat. Oncol. Biol. Phys., vol. 86, no. 5, pp. 986992, Aug. 2013.

DOI: 10.1016/j.jjrobp.2013.04.041

PMid: 23845847

PMCid: PMC 3710733

12. C. I. Rios et al., "Building the strategic national stockpile through the NIAID Radiation Nuclear Countermeasures Program," Drug Dev. Res., vol. 75, no. 1, pp. 23-28, Feb. 2014

DOI: $10.1002 /$ ddr.21163

PMid: 24648046

13. Guide for the care and use of laboratory animals, 8th ed., ILAR, Washington, (DC), USA, 2011, pp. 1-154.
Retrieved from:

https://grants.nih.gov/grants/olaw/Guide-for-the-Careand-Use-of-Laboratory-Animals.pdf Retrieved on: Jan. 4, 2017

14. J. S. Bradley, M. A. Jackson, "The use of systemic and topical fluoroquinolones," Pediatrics, vol. 128, no. 4, pp. 1034-1045, Oct. 2011.

DOI: $10.1542 /$ peds.2011-1496 PMid: 21949152

15. P. D. Tamma et al., "Combination therapy for treatment of infections with gram-negative bacteria," Clin. Microbiol. Rev., vol. 25, no. 3, pp. 450-470, Jul. 2012.

DOI: 10.1128/CMR.05041-11 PMid: 22763634

PMCid: PMC3416487

16. AVMA guidelines for the euthanasia of animals, AVMA, Schaumburg (IL), USA, 2013, p. 50.

Retrieved from:

https://www.avma.org/KB/Policies/Documents/euthan asia.pdf

Retrieved on: Jan. 4, 2017

17. A. L. Carsten, "Acute lethality - the hematopoietic syndrome in different species," in Response of different species to total body irradiation, vol. 10, Lieden, Netherlands: Brill Publishers, 1984, ch. 1, pp. 59-86.

DOI: 10.1007/978-94-009-6048-0_5

18. M. Moroni et al., "Evaluation of the gamma-H2AX assay for radiation biodosimetry in a swine model," Int. J. Mol. Sci., vol. 14, no. 7, pp. 14119-14135, Jul. 2013.

DOI: $10.3390 /$ ijms140714119

PMid: 23880859

PMCid: PMC3742235

19. T. Johansen et al., "The obese Göttingen minipig as a model of the metabolic syndrome: dietary effects on obesity, insulin sensitivity, and growth hormone profile," Comp. Med., vol. 51, no. 2, pp. 150-155, Apr. 2001.

PMid: 11922179

20. H. L. Abrams, "Influence of Age, Body Weight, and Sex on Susceptibility of Mice to the Lethal Effects of X-radiation,” Exp. Biol. Med., vol. 76, pp. 729-732, Apr. 1951.

DOI: $10.3181 / 00379727-76-18610$

21. D. C. Jones et al., "Age at x-irradiation and acute mortality in the adult male rat," Rad. Res., vol. 38, no. 3 , pp. 614-621, Jun. 1969.

DOI: $10.2307 / 3572620$ PMid: 5790124

22. B. Patel et al., "Mobilisation of haematopoietic stem cells in paediatric patients, prior to autologous transplantation following administration of plerixafor and G-CSF," Pediatr. Blood Cancer, vol. 62, no. 8, pp. 1477-1480, Aug. 2015.

DOI: $10.1002 / \mathrm{pbc} .25467$ PMid:25755177

23. A. M. Farese et al., "Filgrastim improves survival in lethally irradiated nonhuman primates," Rad. Res., vol. 179, no. 1, pp. 89-100, Jan. 2013.

DOI: $10.1667 / R_{3049.1}$

PMid: 23210705 PMCid: PMC4562422

24. M. F. Ozkaynak et al., "Randomized comparison of antibiotics with and without granulocyte colonystimulating factor in children with chemotherapyinduced febrile neutropenia: A report from the children's Oncology group," Pediatr. Blood Cancer, vol. 45, no. 3, pp. 274-280, Sep. 2005. DOI: $10.1002 / \mathrm{pbc} .20366$ PMid: 15806544 